\title{
Cystic fibrosis and the relevance of the whole-body vibration exercises in oscillating platforms: a short review
}

\author{
Adalgisa leda Maiworm ${ }^{1}$, Milena B. Monteiro ${ }^{2}$ Sebastião D. Santos-Filho ${ }^{3 *}$, Agnaldo J. Lopes ${ }^{4}$, \\ Leandro Azeredo ${ }^{5}$, Sotiris Missailidis ${ }^{6}$, Pedro J. Marín ${ }^{7,8}$, Mario Bernardo-Filho ${ }^{2,9}$ \\ ${ }^{1}$ Hospital Universitário Pedro Ernesto, Setor de Fisioterapia, Universidade do Estado do Rio de Janeiro, Rio de Janeiro, Brazil; \\ ${ }^{2}$ Clínica de Fisioterapia e Biomedicina, Rio de Janeiro, Brazil; \\ ${ }^{3}$ Universidade do Estado do Rio de Janeiro, Instituto de Biologia Roberto Alcantara Gomes, Departamento de Biofísica e Biometria, \\ Rio de Janeiro, Brazil; ${ }^{*}$ Corresponding Author: santos-filho@uerj.br \\ ${ }^{4}$ Departamento de Pneumologia e Coordenação do Centro de Referência de Fibrose Cística em Adultos do Estado do Rio de Janeiro, \\ Universidade do Estado do Rio de Janeiro, Rio de Janeiro, Brazil; \\ ${ }^{5}$ Hospital da Polícia Militar, Niterói, Brazil; \\ ${ }^{6}$ The Open University, Milton Keynes, UK; \\ ${ }^{7}$ Laboratory of Physiology, European University Miguel de Cervantes, Valladolid, Spain; \\ ${ }^{8}$ Research Center on Physical Disability, ASPAYM, Castilla y León, Spain; \\ ${ }^{9}$ Coordenadoria de Pesquisa, Instituto Nacional do Câncer, INCa, Rio de Janeiro Brazil.
}

Received 29 May 2011; revised 20 July 2011; accepted 8 September 2011.

\section{ABSTRACT}

Objective: The aim is to present a review about the whole-body vibration (WBV) exercises in the management of cystic fibrosis (CF). Methods: Computerized literature searches were performed using the PubMed databases. Results: A strong interest in studies, considering the number of publications, involving CF and lung was found. Although, the exercises and physical activities seem to have a high relevance in the management of the patients with CF, the number of publications involving these procedures is limited. Moreover, a modality of exercise involving WBV seems to be poorly utilized by the patients with CF and only two papers were found with positive actions. Conclusion: Oscillating platform is a promising technique to manage patients with CF. Moreover, as 1) the use of the oscillating platforms is inexpensive, 2) the vibration exercise requires less technical abilities as compared to the performance of conventional resistance training and 3) positive clinical findings have been noticed with the use of WBV in CF, it is suggested to implement the studies involving the application of the exercises with WBV in oscillating platforms to manage the patients with $C F$.

Keywords: Cystic fibrosis; whole body vibration;
PubMed

\section{INTRODUCTION}

Cystic fibrosis (CF), also known as mucoviscidosis [1], is the most common fatal inherited chronic disease most frequent in white populations [2-4] and affects one in every 2,500 Europeans, with a similar incidence in Brazil [1,3,5]. Furthermore, CF Foundation [6] considers that $\mathrm{CF}$ affects the lungs and digestive system of about 30,000 children and adults in the United States $(70,000$ worldwide).

CF is a congenital and multi-systemic disease and there are several clinic manifestations due to the cystic fibrosis genetic characteristic with more than 1000 acknowledged mutations so far, though there is always a triad of symptoms in the pulmonary and digestive tracts and in the sweat glands [4]. However, the primary clinical manifestations usually occur in the lungs and include cough, excess sputum, shortness of breath and respiratory muscle fatigue, resulting in progressive lung damage and eventual death from respiratory failure [2].

$\mathrm{CF}$ also affects the mucus production of exocrine glands, which become thick and obstruct the channels in which it is secreted. This manifestation can occur in numerous epithelial cells including sweat, pancreatic and bile ducts, airways, intestinal and deferent vessels [4].

The CF can be considered a stigmatizing disease because it marks those who live with it. People with CF can have a variety of symptoms, including 1 ) very salty- 
tasting skin, 2) persistent coughing, at times with phlegm, 3) frequent lung infections, 4) wheezing or shortness of breath, 5) poor growth/weight gain in spite of a good appetite; and 6) frequent greasy, bulky stools or difficulty in bowel movements [6]. Furthermore, there is body deformity caused by the evolution of the disease, which marks the body in a characteristic way, including producing a barrel chest and clubbing of the feet. Additionally, there are daily and constant care rituals, which often times impede children and adolescents from performing activities common to their age group [7].

The therapies for CF involve drugs to help clear the thick CF fibrosis mucus from airways, reduce inflammation and aerosolized antibiotics for CF. People with CF do airway clearance techniques to loosen and get rid of the mucus from the lungs. Clearing mucus helps to reduce the severity of lung infections and improve lung function. Inhaled drugs are commonly used in CF care be- cause they reach the airways quickly and easily. Antibiotics are used to fight infection-causing bacteria. Infections are common in the lungs of people with CF, so antibiotics are an important part of regular care $[3,6]$. In the general population, the nutrition needs change with age, however, a special attention would be addressed for people with CF. Children and teens with CF need extra calories to grow and develop. Everyone with $\mathrm{CF}$, no matter their age, need good nutrition to stay strong against lung infections and other challenges [6].

Another important procedure to be used in the patients with CF is the exercises. Not surprisingly, CF physiotherapists and people with CF need clear information and guidance on how to incorporate exercise into an already stressful and arduous daily regime. In addition, exercises and physical activities seem to be important to contribute to increase the life expectancy of the patients with CF [2,8-10].

There is evidence of the benefit for the patients with CF in increasing the participation in the daily activities, improving the quality of life and reducing the hospital admissions. Bradley et al. [11] demonstrated that exercises have positive effects on exercise capacity, strength and lung function in CF. It is generally accepted that exercise tolerance is linked to a decline in lung disease and it is an independent predictor of survival [12-15].

Vibrations, defined as an oscillatory motion, can be generate in oscillating platforms and transmitted, in general, by the feet to whole body of a person [16]. The frequency and the amplitude of the sinusoidal vibration can be manipulated by the professional that is superviseing the clinical procedure. The duration of the work, as well as, the time to rest, the number of sets in a session, the number of sessions is also controlled. All these conditions depend on, mainly, the clinical and physical con- ditions of the patient [17,18]. Moreover, Marin et al. [19] have also studied the neuromuscular activity during whole-body vibration (WBV) of different amplitudes, besides the footwear conditions.

The mechanisms responsible for the WBV benefits are not conclusive, however Santos-Filho et al. [17] have suggested that these effects are probably related to direct and indirect actions. Prisby et al. [20] have suggested that the indirect effects might to be associated with the neuroendocrine system. Whole body mechanical vibration on the muscle performance would be due to the induction of a myotatic reflex contraction referred as the tonic vibration reflex [21,22]. Furthermore, some authors have described that, potentially, repeated muscle contractions might exert endocrine and/or metabolic effects [23]. Furthermore, WBV would act through repetitive sensorial-motor stimulation [24].

Authors have demonstrated that the exercises due to the WBV improve walking function [25], muscle strength [27], bone mineral density [27], cardiovascular fitness [26] and body balance [28]. Moreover, the health-related quality of life is increased and the fall risk is decreased. Improvement of gait and balance with WBV has been shown in a population of nursing home residents [29].

Some works considering the use of the whole body vibrations produced in oscillating platforms to manage patients with CF have been reported [30,31].

Putting together the findings reported in the literature, investigations about the effectiveness of the action of the vibration produced in oscillating platforms that is a low cost physiologic strategy to treat CF would be welcome.

Citations found in the PubMed have been used as tool to obtain various scientific informations [32,33]. Moreover, to our knowledge no previous suitable revisions have been published involving WBV and CF. As the relevance of exercise in the management of CF has been well recognized [34], the aim of this work is to present a suitable review about the published papers found in the PubMed databank in which there are information about the use of the WBV exercises in the management of patients with CF.

\section{METHODS}

PubMed is a service of the United States National Library of Medicine of the National Institutes of Health [35]. PubMed is a databank that comprises more than 19 million citations for biomedical articles from MEDLINE and life science journals. These citations may include links to full-text articles from PubMed Central or publisher web sites.

Strategy to search in the PubMed

The number of publications (NP) was determined 
using the follow strategy. 1) All the papers were searched in the PubMed on April $21^{\text {st }} 2010$. 2) A search involving the terms "cystic fibrosis" and mucoviscidosis was performed considering a) mucoviscidosis, b) mucoviscidosis or "cystic fibrosis" and c) cystic fibrosis. 3) A search was performed using the keywords a) "cystic fibrosis", b) "cystic fibrosis" and lungs, c) "cystic fibrosis" and kidneys, d) "cystic fibrosis" and stomach, e) "cystic fibrosis" and liver, f) "cystic fibrosis" and "sweat glands”, g) "cystic fibrosis" and pancreas and h) "cystic fibrosis" and heart. 4) A search involving cystic fibrosis and gender was carried out using the keywords a) "cystic fibrosis" and male, b) "cystic fibrosis" and female. 5) A search involving cystic fibrosis and the human development was carried out using the keywords a) "cystic fibrosis" and adult, b) "cystic fibrosis" and children, c) "cystic fibrosis" and adolescents, d) "cystic fibrosis" and older and e) "cystic fibrosis" and pregnancy. 5) A search involving CF and some possible types of treatments was carried out using the keywords a) "cystic fibrosis" and antibiotics, b) "cystic fibrosis" and medications, c) "cystic fibrosis" nutrition, d) "cystic fibrosis" and exercises and e) "cystic fibrosis" and physical activity.

The selected publications involving "whole body vibration" and CF were read and considered to be discussed in this work.

\section{RESULTS}

The number of publications found in the PubMed involving the terms "cystic fibrosis" and mucoviscidoses are shows in Table $\mathbf{1}$ and it is almost the same.

Table 2 shows the number of publications involving "cystic fibrosis" and some organs that could be related with some consequences of the "cystic fibrosis". The publications involving CF present the highest number of scientific citations. Although, an interest it is found in the studies related to the pancreas, heart and liver.

Table 3 shows the number of publications involving "cystic fibrosis" and the gender. The number of publications is practically the same, although the number of publications related the sex is slightly bigger for the female, about $50.5 \%$.

Table 4 shows the number of publications involving "cystic fibrosis" and steps of the human development. There is a strong scientific interest in investigations relating to CF and children. However, an important interest is found in the studies with adult and adolescents. A limited interest is found to the works involving older and cystic fibrosis.

Table 5 shows the number of publications involving "cystic fibrosis" and some possible modalities of treatments. The use of antibiotics has had a strong interest of the scientific community as an option to treat the symp- toms associated with CF. A small number of publications are found to exercises and physical activity. Moreover, only the authors of 2 papers have used the WBV to manage patients with cystic fibrosis.

The two papers that have described the use of the WBV to treat patients with CF were read and analyzed in Tables 6 and 7. In the Table 6 are shown information about the device of the oscillating platform, the subjects (number, sex and age), the frequency and the amplitude used in the oscillating platforms. In the Table 7 are shown information about the conditions of protocols used in the treated and control groups, as well as the clinical findings.

Table 1. Number of publications found in the PubMed involving "cystic fibrosis" and mucoviscidosis.

\begin{tabular}{lc}
\hline Search & Number of publications \\
\hline “cystic fibrosis” & 31,938 \\
Mucoviscidosis & 32,843 \\
“cystic fibrosis” or mucoviscidosis & 32,843 \\
\hline
\end{tabular}

Table 2. Number of publications found in the PubMed involving "cystic fibrosis” and some organs.

\begin{tabular}{lc}
\hline Search & Number of publications \\
\hline “cystic fibrosis” and lungs & 7976 \\
“cystic fibrosis” and pancreas & 1786 \\
"cystic fibrosis” and kidneys & 750 \\
“cystic fibrosis” and heart & 1264 \\
“cystic fibrosis” and "sweat glands” & 228 \\
“cystic fibrosis” and stomach & 128 \\
"cystic fibrosis” and liver & 1290 \\
\hline
\end{tabular}

Table 3. Number of publications found in the PubMed involving "cystic fibrosis" and the gender.

\begin{tabular}{lc}
\hline Search & Number of publications \\
\hline “cystic fibrosis” and male & 10,888 \\
"cystic fibrosis” and female & 11,111 \\
\hline
\end{tabular}

Table 4. Number of publications found in the PubMed involving "cystic fibrosis" and some steps of the human development.

\begin{tabular}{lc}
\hline Search & Number of publications \\
\hline “cystic fibrosis” and children & 11,321 \\
“cystic fibrosis” and adolescents & 7664 \\
“cystic fibrosis” and adult & 8904 \\
“cystic fibrosis” and older & 547 \\
“cystic fibrosis” and pregnancy & 1353 \\
\hline
\end{tabular}


Table 5. Number of publications found in the PubMed involving "cystic fibrosis” and possible modalities of treatments.

\begin{tabular}{lc}
\hline Search & Number of publications \\
\hline “cystic fibrosis” and antibiotics & 3105 \\
“cystic fibrosis” and medications & 1171 \\
“cystic fibrosis” and exercises & 557 \\
“cystic fibrosis” and nutrition & 967 \\
“cystic fibrosis” and “physical activity” & 91 \\
“cystic fibrosis” and “whole body vibration” & 2 \\
\hline
\end{tabular}

Table 6. Information about the device of the oscillating platform, the subjects, the frequency and the amplitude used in the oscillating platforms.

\begin{tabular}{|c|c|c|c|c|}
\hline Reference & $\begin{array}{c}\text { Device of the } \\
\text { platform }\end{array}$ & $\begin{array}{c}\text { Number of } \\
\text { subjects/sex/age }\end{array}$ & Frequency & Amplitude \\
\hline $\begin{array}{l}\text { Rietschel } \\
\text { et al., } 2008\end{array}$ & $\begin{array}{l}\text { Galileo 2000, } \\
\text { Novotec } \\
\text { GmbH, } \\
\text { Pforzheim, } \\
\text { Germany }\end{array}$ & $\begin{array}{c}10 \text { clinically stable } \\
\text { patients ( } 3 \text { males and } \\
7 \text { female, } 24 \text { - } 47 \\
\text { years }\end{array}$ & $20-25 \mathrm{~Hz}$ & $0.5 \mathrm{~mm}$ \\
\hline $\begin{array}{l}\text { Roth et al., } \\
2008\end{array}$ & $\begin{array}{c}\text { GalileoTM } \\
\text { platform } \\
\text { (Novotec } \\
\text { Medical, } \\
\text { Pforzheim, } \\
\text { Germany) }\end{array}$ & $\begin{array}{l}11 \text { adult patients, } \\
21 \text { - } 41 \text { years old }\end{array}$ & $\begin{array}{c}12 \mathrm{~Hz} \text { and } \\
26 \mathrm{~Hz}\end{array}$ & $\begin{array}{c}\text { No } \\
\text { specified }\end{array}$ \\
\hline
\end{tabular}

Table 7. Information about the conditions of protocols used in the treated and control groups and the clinical findings.

\begin{abstract}
After 3 months of WBV all parameters in the CRT significantly improved: chair-rising The training schedule consisted of 3 ses- time, maximal force, maximal power and sions of 3 min twice a day, 5 days per week velocity. The peak jump force and velocity for 3 months. The patients were standing in of the two-leg jump significantly improved. Rietschel the platform with flexed knees (20 - 45 Parameters in the one-leg jump as well as et al, degrees). In addition, exercises were per- maximal isometric grip force showed no 2008 formed (trunkrotation and trunklateral flex- significant improvement. Weight and body ions for abdominal, trunkextension for mass index showed a slightly positive trend abdominal and trunkflexion for mobility of whereas $\mathrm{FEV}_{1}$ and FVC did not signifispine)
\end{abstract}

These results demonstrate that WBV can improve muscle function in CF patients.
On five days per week, one unit of six minutes duration at a frequency of $12 \mathrm{~Hz}$ was carried out to improve the range of motion of the thorax, spine and extremities.

On three days per week, one unit of six minutes duration at frequencies up to $26 \mathrm{~Hz}$ (according to the individual patient's capabilities) was performed including additional weights of up to nine kilograms to increase muscle power and force.

During each unit $(12 \mathrm{~Hz}$ and $26 \mathrm{~Hz}$ ), the patient performed standardized manoeuvers including leg bends, trunk bends and extension and rotation of the trunk while standing on the device.
Cardiovascular monitoring did not show any critical drop in oxygen saturation or blood pressure. Lung function remained relatively constant with a median $\mathrm{FEV}_{\text {}}$ change [\% of norm] of $-3.1 \%$ (range -7 20). Trabecular density at the spine and parameters of bone density and geometry at the radius and tibia did not show consistent changes. A median decrease of $-0.3 \%$ $(-31.0$ - 17.9) for muscle force and a median increase of $4.7 \%$ (-16.4 - 74.5) for muscle power and $6.6 \%(-0.9-48.3)$ for velocity was noted in the two-leg jump. In the one-leg jump, a median increase of $6.7 \%$ (-8.5 - 24.3) for muscle force was measured.
Whole body vibration was well tolerated in the majority of the study participants. Most patients were able to increase peak force in the one-leg jump.

In the two-leg jump, velocity and muscle power increased with equal or decreased muscle force. This may indicate an improvement in neuromuscular and intramuscular co-ordination (and therefore efficiency) with less muscle force necessary to generate the same power. 


\section{DISCUSSION}

CF can be considered a stigmatizing disease, which marks the body of the patient, including producing a barrel chest and clubbing of the feet. Moreover, there are daily and constant care rituals, which can limit the participation of these patient in common daily activities that are common to their age [7]. CF is also known as mucoviscidosis [7] and in a search in the databank PubMed, it is found no difference in the number of publications using the keywords "cystic fibrosis" or mucoviscidosis, alone or together (Table 1).

The primary symptoms of the CF involve the lungs however an important increase in the life expectancy [2] has been noticed. In consequence, co-morbidities affecting organ systems other than the lungs have been reported. The data shown in the Table 2 revealed a strong interest in studies, considering the number of publications, involving $\mathrm{CF}$ and lung as it was expected. However, an important scientific interest was found to investigations involving CF and pancreas, heart or liver that could be justified due to the increase in the life expectancy. Moreover, the predicted median age of survival for a person with $\mathrm{CF}$ is more than 37 years $[2,3,6]$.

Within the CF population, there are some evidences that females with CF may be less active than males [13, 14]. These differences in physical activity levels between males and females may contribute to the greater decline in lung function in female with CF [36]. This finding could justify the statement of Courtney et al. [37] that female gender is an important predictor of increased risk of death, although the mechanisms causing this are unclear. However, the prevalence of potential risk factors is not higher among females than males [38] and this fact could justify the almost the same number of publications in CF in male and female (Table 3).

The prognosis of patients with CF has improved substantially over the last three decades [37]. Although the majority of the patients still die in early adulthood [39], the life expectancy for patients has increased from 31 to 37 years over the last decade [40]. Nick et al. [41], have reported that long-term CF survivors (age $>40$ years) are a growing population comprised of both patients diagnosed with classic manifestations in childhood, and non-classic phenotypes typically diagnosed as adults. Little is known concerning disease progression and outcomes in these cohorts. These findings and statements aid to explain the elevated number of publications involving $\mathrm{CF}$ and children, as well as an important number of publications involving adolescents and adults (Table 4). A UK model predicting that a child born with CF today will typically live to be 50 years or more seems to be realistic [39] and this model aids to justify the number of studies with CF and pregnancy (Table 4).
The daily treatment burden of CF is high and people may spend up to $2 \mathrm{~h}$ per day on a wide range of CF therapies, including taking medications and undergoing physiotherapy treatments such as regular airway clearance and exercise. The therapy with antibiotics is highly important and this fact can be reinforced with the strong number of publication with these drugs when a comparison with other therapy is done. Naturally the studies with medications in general are welcome and an important number of publications is found. The benefits of maintaining good nutrition for the patients with CF have been reported by several authors $[3,42,43]$ and this fact could justify the elevated number of publications involving CF and nutrition (Table 5).

Although, the exercises and physical activities [2,34], seem to have a high relevance in the management of the patients with CF, the number of publications involving these procedures is still limited and the general adherence to treatments in CF is challenging and the adherence to exercise is lower compared with other treatments $[8,44,45]$. Moreover, a modality of exercise involving whole body vibrations seems to be poorly utilized by the patients with CF and only two papers were found in the PubMed databank (Table 5).

Vibrations produced in the oscillating platforms and transmitted to be whole body have been considered as a kind of exercise that is useful and safe to be used with elderly $[26,28]$, as well as in patients with some neuronlogical disorders $[24,25]$. Furthermore, this kind of exercises has been considered to be of a low cost physiologic strategy [46]. Furthermore, the vibration exercise requires less technical abilities as compared to the performance of correct conventional resistance training [21, 24].

The two papers found after a search in the PubMed using the keywords "cystic fibrosis" and "whole body vibration" have reported positive actions in the patients with cystic fibrosis (Table 6). Although the protocols, as well as the parameters used by Rietschel et al. [30] and Roth et al. [31] are well different, the conclusion of both works is that the exercises in oscillating platforms are suitable to manage patients with CF.

It is highly relevant the development of clinical procedures to the management of patients with CF. Oscillating platform is a promising technique to improve muscle power and to a certain extent force in patients with CF. The advantage is that the amount of time that has to be spent for the training is fairly minimal. Moreover, as 1) the use of the oscillating platforms is very inexpensive [46] and 2) positive clinical findings have been noticed with the use of whole body vibration in patients with CF, it is suggested to implement the studies involving the application of the exercises with whole 
body vibration in oscillating platforms to manage the patients with $\mathrm{CF}$.

\section{ACKNOWLEDGEMENTS}

We thank CNPq (Conselho Nacional de Desenvolvimento Científico e Tecnológico), FAPERJ (Fundação de Amparo à Pesquisa do Rio de Janeiro), UERJ (Universidade do Estado do Rio de Janeiro) and INCa (Instituto Nacional do Câncer).

\section{REFERENCES}

[1] Pizzignacco, T.M.P., Mello, D.F. and de Lima, R.A.G. (2010) Stigma and cystic fibrosis. Revista Latino Americanda de Enfermagem, 18, 139-142. doi:10.1590/S0104-11692010000100021

[2] Moran, F. and Bradley, J. (2010) Incorporating exercise into the routine care of individuals with cystic fibrosis: Is the time right? Expert Review of Respiratory Medicine, 4, 139-142. doi:10.1586/ers.10.18

[3] O’Sullivan B.P. and Freedman, S.D. (2009) Cystic fibrosis. Lancet, 373, 1891-1904. doi:10.1016/S0140-6736(09)60327-5

[4] Davis, P.B. (2006) Cystic fibrosis since 1938. American Journal of Respiratory and Critical Care Medicine, 173, 475-482. doi:10.1164/rccm.200505-8400E

[5] Ribeiro, D.J., de Ribeiro, M.A.G.O. and Ribeiro, A.F. (2002) Controvérsias na fibrose cística: Do pediatra ao especialista. In Portuguese, Journal of Pediatrics, 78, 171-186.

[6] Cystic Fibrosis Foundation. (2010). http://www.cff.org.

[7] Pizzignacco, T.M.P. and de Lima, R.A.G. (2006) Socialization of children and adolescents with cystic fibrosis: A support for nursing care. Revista Latino Americanda de Enfermagem, 14, 569-577. doi:10.1590/S0104-11692006000400015

[8] Sawicki, G., Sellers, D. and Robinson, W. (2009) High treatment burden in adults with cystic fibrosis: Challenges to disease self management. Journal of Cystic Fibrosis, 9, 91-96. doi:10.1016/j.jcf.2008.09.007

[9] Myers, L.B. (2009) An exploratory study investigating factors associated with adherence to chest physiotherapy and exercise in adults with cystic fibrosis. Journal of Cystic Fibrosis, 8, 425-427. doi:10.1016/j.jcf.2009.08.005

[10] Selvadurai, H.C., Blimkie, C.J., Cooper, P.J., Mellis, C.M. and Van Asperen, P.P. (2004). Gender differences in habitual activity in children with cystic fibrosis. Archives of Disease in Childhood, 89, 928-933. doi:10.1136/adc.2003.034249

[11] Bradley, J.M. and Moran, F. (2008). Physical training for cystic fibrosis. Cochrane Database of Systematic Reviews, 1, CD002768.

[12] Nixon, P.A., Orenstein, D.M., Kelsey, S.F. and Doerschuk, C.F. (1992) The prognostic value of exercise testing in patients with cystic fibrosis. The New England Journal of Medicine, 327, 1785-1788. doi:10.1056/NEJM199212173272504

[13] Klijn, P.H., Oudshoorn, A., van der Ent, C.K., van der Net, J., Kimpen, J.L. and Helders, P.J. (2004) Effects of anaerobic training in children with cystic fibrosis: A ran- domised controlled study. Chest, 125, 1299-1305. doi:10.1378/chest.125.4.1299

[14] Pianosi, P., LeBlanc, J. and Almudevar, A. (2005a) Peak oxygen uptake and mortality in children with cystic fibrosis. Thorax, 60, 50-54. doi:10.1136/thx.2003.008102

[15] Pianosi, P., LeBlanc, J. and Almudevar, A. (2005b) Relationship between $\mathrm{FEV}_{1}$ and peak oxygen uptake in children with cystic fibrosis. Pediatric Pulmonology, 40, 324-329. doi:10.1002/ppul.20277

[16] Bressel, E., Smith, G., and Branscomb, J. (2009). Transmission of whole vibration in children while standing. Clinical Biomechanics, 25, 181-186. doi:10.1016/j.clinbiomech.2009.10.016

[17] Santos-Filho, S.D., Meyer, P.F., Ronzio, O.A., Bonelli, L., Fonseca, A.S. and Bernardo-Filho, M. (2010) Whole body vibration exercise: What do you know about scientific interest? FIEP Bulletin, 80, 875-878.

[18] Cardinale, M. and Wakeling, J. (2005) Whole body vibration exercises: Are vibrations good for you? British Journal of Sports Medicine, 39, 585-589. doi:10.1136/bjsm.2005.016857

[19] Marín, P.J., Bunker, D., Rhea, M.R. and Ayllon, F.N. (2009) Neuromuscular activity during whole-body vibration of different amplitudes and footwear conditions: Implications for prescription of vibratory stimulation. The Journal of Strength \& Conditioning Research, 23, 2311-2316. doi:10.1519/JSC.0b013e3181b8d637

[20] Prisby, R.D., Lafage-Proust, M., Malaval, L., Belli. A. and Vico, L. (2008) Effects of whole body vibration on the skeleton and other organ systems in man and animal models: What we know and what we need to know. Ageing Research Reviews, 7, 319-329. doi:10.1016/j.arr.2008.07.004

[21] Torvinen, S., Sievanen, H., Jarvinen, T.A., Pasanen, M., Kontulainen, S. and Kannus, P. (2002) Effect of 4-min vertical whole body vibration on muscle performance and body balance: A randomized cross-over study. International Journal of Sports Medicine, 23, 374-379. doi:10.1055/s-2002-33148

[22] Romaiguere, P., Vedel, J.P. and Pagni, S. (1993) Effects of tonic vibration reflex on motor unit recruitment in human wrist extensor muscle. Brain Research, 602, 32-40. doi:10.1016/0006-8993(93)90237-H

[23] Di Loreto, C., Ranchelli, A., Lucidi, P., Murdolo, G., Parlanti, N. and De Cicco, A., et al. (2004) Effects of whole-body vibration exercise on the endocrine system of healthy men. Journal of Endocrinological Investigation, 27, 323-327.

[24] Ebersbach, G., Edler, D., Kaufhold, O. and Wissel, J. (2008) Whole body vibration versus conventional physiotherapy to improve balance and gait in Parkinson's disease. Archives of Physical Medicine and Rehabilitation, 89, 399-403. doi:10.1016/j.apmr.2007.09.031

[25] Ness, L.L. and Field-Fote, E.C. (2009) Whole-body vibration improves walking function in individuals with spinal cord injury: A pilot study. Gait Posture, 30, 436-440. doi:10.1016/j.gaitpost.2009.06.016

[26] Bogaerts, A.C.G., Delecluse, C., Claessens, A., Troosters, T., Boonen, S. and Verschueren, S.M.P. (2009) Effects of whole body vibration training on cardiorespiratory fitness and muscle strength in older individuals (a 1-year randomised controlled trial). Age Ageing, 38, 448-454. 
doi:10.1093/ageing/afp067

[27] Gusi, N., Raimundo, A. and Leal, A. (2006) Low-frequency vibratory exercise reduces the risk of bone fracture more than walking: A randomized controlled trial. BMC Musculoskelet Disord, 7, 92. doi:10.1186/1471-2474-7-92

[28] Kawanabe, K., Kawashima, A., Sashimoto, I., Takeda, T., Sato, Y. and Iwamoto, J. (2007) Effect of whole body vibration exercise and muscle strengthening, balance, and walking exercises on walking ability in the elderly. The Keio Journal of Medicine, 56, 28-33. doi:10.2302/kjm.56.28

[29] Bruyere, O., Wuidart, M.A., Di Palma, E., Gourlay, M., Ethgen, O., Richy, F., et al. (2005) Controlled whole body vibration to decrease fall risk and improve healthrelated quality of life of nursing home residents. Archives of Physical Medicine and Rehabilitation, 86, 303-307. doi:10.1016/j.apmr.2004.05.019

[30] Rietschel, E., van Koningsbruggen, S., Fricke, O., Semler, O. and Schoenau, E. (2008) Whole body vibration: A new therapeutic approach to improve muscle function in cystic fibrosis? International Journal of Rehabilitation Research, 31, 253-256. doi:10.1097/MRR.0b013e3282fb783d

[31] Roth, J., Wust, M., Rawer, R., Schnabel, D., Armbrecht, G., Beller, G. et al. (2008) Whole body vibration in cystic fibrosis: A pilot study. Journal of Musculoskeletal and Neuronal Interactions, 8, 179-187.

[32] Santos-Filho, S.D., Sohaku, R.C.B., Pereira, F.A.O., Senna-Fernandes, V., França, D., Guilhon, S., et al. (2004) Traditional medicine: An evaluation of the interest of the publication of scientific papers about moxibustion. The American Journal of the Medical Sciences, 4, 59-62. doi:10.3923/jms.2004.59.62

[33] Winchester, D.E., Wymer, D.C., Shifrin, R.Y., Kraft, S.M. and Hill, J.A. (2010) Responsible use of computed tomography in the evaluation of coronary artery disease and chest pain. Mayo Clinic Proceedings, 85, 358-364. doi:10.4065/mcp.2009.0652

[34] Bott, J., Blumenthal, S., Buxton, M., Ellum, S., Falconer, C., Garrod, R., et al. (2009) Guidelines for the physiotherapy management of the adult, medical, spontaneously breathing patient. Thorax, 64, 1-51. doi:10.1136/thx.2008.110726

[35] PubMed. (2010). http://www.ncbi.nlm.nih.gov/PubMed/.

[36] Schneiderman-Walker, J., Wilkes, D.L., Strug, L., Lands, L.C., Pollock, S.L. and Selvadurai, H.C., et al. (2005)
Sex differences in habitual physical activity and lung function decline in children with cystic fibrosis. Journal of Pediatrics, 147, 321-326. doi:10.1016/j.jpeds.2005.03.043

[37] Courtney, J.M., Bradley, J., Mccaughan, J., O’connor, T.M., Shortt, C., Bredin, C.P., et al. (2007) Predictors of mortality in adults with cystic fibrosis. Pediatric Pulmonology, 42, 525-532. doi:10.1002/ppul.20619

[38] Rosenfeld, M., Davis, R., FitzSimmons, S., Pepe, M. and Ramsey, B. (1997) Gender gap in cystic fibrosis mortality. American Journal of Epidemiology, 145, 794-803.

[39] Dodge, J.A., Lewis, P.A., Stanton, M. and Wilsher, J. (2007) Cystic fibrosis mortality and survival in the UK: 1947-2003. European Respiratory Journal, 29, 522-526. doi:10.1183/09031936.00099506

[40] Cystic Fibrosis Foundation. (2007) Annual data report to the center directors. http://www.cff.org.

[41] Nick, J.A., Chacon, C.S., Brayshaw, S.J., Jones, M.C., Barboa, C.M., St Clair, C.G., et al. (2010) Effects of gender and age of diagnosis on disease progression in long-term cystic fibrosis survivors. American Journal of Respiratory and Critical Care Medicine, 182, 614-626. doi:10.1164/rccm.201001-0092OC

[42] Peterson, M.L., Jacobs, D.R. and Milla, C.E. (2003) Longitudinal changes in growth parameters are correlated with changes in pulmonary function in children with cystic fibrosis. Pediatrics, 112, 588-592. doi:10.1542/peds.112.3.588

[43] Corey, M., McLaughlin, F.J., Williams, M. and Levison, H. (1988) A comparison of survival, growth, and pulmonary function in patients with cystic fibrosis in Boston and Toronto. Journal of Clinical Epidemiology, 41, 583-591. doi:10.1016/0895-4356(88)90063-7

[44] White, W., Stiller, K. and Hansel, N. (2007) Adherence of adult cystic fibrosis patients with airway clearance and exercise regimes. Journal of Cystic Fibrosis, 6, 163-170. doi:10.1016/j.jcf.2006.06.006

[45] Myers, L.B. and Myers, F. (1999) The relationship between control beliefs and self-reported adherence in adults with cystic fibrosis. Psychology, Health \& Medicine, 4, 387- 392. doi:10.1080/135485099106135

[46] Alentorn-Geli, E., Moras, G., Padilla, J., Fernandez-Sola, J., Bennett, R.M., Lazaro-Haro, C., et al. (2009) Effect of acute and chronic whole-body vibration exercise on serum insulin-like growth factor-1 levels in women with fibromyalgia. Journal of Alternative and Complementary Medicine, 15, 573-578. doi:10.1089/acm.2008.0366 